[16] T. Keviczky, F. Borrelli, and G. J. Balas, "Decentralized receding horizon control for large scale dynamically decoupled systems," Automatica, vol. 42, pp. 2105-2115, 2006.

[17] A. Sarwar, "Spatiotemporal Systems: Gradual Variations, Indentification, Adaptation and Robustness," Ph.D. dissertation, University of Illinois at Urbana-Champaign, Urbana, IL, 2009.

[18] A. P. Featherstone and R. D. Braatz, "Control relevant identification of sheet and film processes," in Proc. Amer. Control Conf., 1996, pp. 2692-2696.

[19] P. Massioni and M. Verhaegen, "Subspace identification of circulant systems," Automatica, vol. 44, pp. 2825-2833, 2008.

[20] A. Sarwar and P. Voulgaris, "Spatially invariant systems: Identification and adaptation," in Proc. 3rd Int. Conf. Appl. Math., Sim., Modelling, Circuits, Syst. Signals, 2009, pp. 208-215.

[21] P. Massioni and M. Verhaegen, "Subspace identification of distributed, decomposable systems," in Proc. IEEE Conf. Decision Control, 2009, pp. 3364-3369.

[22] M. Verhaegen and V. Verdult, Filtering and System Identification-A Least Squares Approach. Cambridge, U.K.: Cambridge Univ. Press, 2007.

[23] M. Ali, S. S. Chughtai, and H. Werner, "Identification of spatially interconnected systems," in Proc. IEEE Conf. Decision Control, 2009, pp. 7163-7168.

[24] R. D'Andrea and G. E. Dullerud, "Distributed control design for spatially interconnected systems," IEEE Trans. Autom. Control, vol. 48, no. 9, pp. 1478-1495, Sep. 2003.

[25] F. Wu, "Distributed control for interconnected linear parameter-dependent systems," IEEE Proc. Control Theory Appl., vol. 150, no. 5, pp. 518-527, Sep. 2003.

[26] J. Fowler and R. D'Andrea, “A formation flight experiment," IEEE Control Syst. Mag., vol. 23, no. 5, pp. 35-43, Oct. 2003.

[27] P. Sharma and C. Beck, "Modelling and distributed control of mobile offshore bases," in Proc. Amer. Control Conf., 2004, pp. 5238-5243.

[28] R. D'Andrea, "Linear matrix inequalities, multidimensional system optimization, and control of spatially distributed systems: An example," in Proc. Amer. Control Conf., 1999, pp. 2713-2717.

[29] P. Dewilde and A. J. V. D. Veen, Time-Varying Systems and Computations. Norwell, MA: Kluwer, 1998.

[30] J. K. Rice and M. Verhaegen, "Distributed control in multiple dimensions: A structure preserving computational technique," IEEE Trans. Autom. Control, vol. 56, no. 3, pp. 516-530, Mar. 2011.

[31] J. K. Rice, "Efficient Algorithms for Distributed Control: A Structured Matrix Approach," Ph.D. dissertation, Delft Univ. Technol., Delft, The Netherlands, 2010.

[32] L. Ljung, "Asymptotic behavior of the extended Kalman filter as a parameter estimator for linear systems," IEEE Trans. Autom. Control, vol. AC-24, no. 1, pp. 36-50, Feb. 1979.

[33] T. McKelvey and A. Helmersson, "System identification using an overparametrized model class-Improving the optimization algorithm," in Proc. IEEE Conf. Decision Control, 1997, pp. 2984-2989.

[34] R. A. Horn and C. R. Johnson, Topics in Matrix Analysis. Cambridge, U.K.: Cambridge Univ. Press, 1991.

[35] J. K. Rice and M. Verhaegen, "Structure preserving model order reduction of heterogeneous 1-d distributed systems," in Proc. ACC, 2009, pp. 4109-4114.

[36] A. Turing, "The chemical basis of morphogenesis," Phil. Trans. Royal Soc. London, Series B, vol. 237, pp. 37-72, 1952.

[37] J. D. Murray, Mathematical Biology: Spatial Models and Biomedical Applications. New York: Springer-Verlag, 2003.

[38] M. S. Yeung, J. Tegner, and J. J. Collins, "Reverse engineering gene networks using singular value decomposition and robust regression," Proc. Nat. Acad. Sci. USA, vol. 99, pp. 6163-6168, 2002.

\section{Stabilization Over Power-Constrained Parallel Gaussian Channels}

\author{
Zhan Shu and Richard H. Middleton
}

\begin{abstract}
This technical note is concerned with state-feedback stabilization of multi-input systems over parallel Gaussian channels subject to a total power constraint. Both continuous-time and discrete-time systems are treated under the framework of $\mathcal{H}_{2}$ control, and necessary/sufficient conditions for stabilizability are established in terms of inequalities involving unstable plant poles, transmitted power, and noise variances. These results are further used to clarify the relationship between channel capacity and stabilizability. Compared to single-input systems, a range of technical issues arise. In particular, in the multi-input case, the optimal controller has a separation structure, and the lower bound on channel capacity for some discrete-time systems is unachievable by linear time-invariant (LTI) encoders/decoders.
\end{abstract}

Index Terms-Channel capacity, $\mathcal{H}_{2}$ control, networked control systems, parallel Gaussian channels, stabilization, transmitted power.

\section{INTRODUCTION}

With the rapid development of communication technology, an increasing number of control systems have integrated communication channels or networks to transmit signals. While simplifying installation, facilitating maintenance, and reducing building costs, the use of communication has also raised several issues and challenges. e.g., transmission delay, packet loss, data rate limit, and quantization. These issues are rarely treated in traditional control system analysis and synthesis, and spurs new developments of control theory [1], [2].

As one of the most important communication constraints, data rate has received much attention recently. By employing information theoretic arguments, Nair and Evans have established a series of results on the relationship between stabilizability and data rate [3]-[5]. LQG type control has been considered in [6], [7]. State estimation and related observability/detectability have been investigated in [8]. A comprehensive survey on control with data rate limit is available in [9]. Although these results are nice, they rely heavily on various information theoretic techniques, which are unfamiliar to most researchers in control society, and the resulting complex encoding/decoding schemes are difficult to analyze in traditional control terms such as performances and robustness. In [10] and subsequent work [11], [12], an alternative framework for control system analysis and synthesis with data rate constraints has been established for single-input and single-output LTI systems with Gaussian communication channels. A highlight of this framework lies in its simplicity and intimate relationship with modern control theory. Naturally, one may wonder whether the proposed framework can cope with multi-input and multi-output systems, and this motivates the present study. As for networked control with other constraints such as quantization, packet dropout, communication delay, we refer readers to [13], [14] and references therein

In this technical note, we study the problem of state-feedback stabilization for multi-input systems over parallel Gaussian channels subject to a total power constraint, and establish necessary/sufficient conditions for stabilizability in terms of inequalities involving unstable plant

Manuscript received July 15, 2010; accepted January 06, 2011. Date of publication April 05, 2011; date of current version July 07, 2011. This work was supported by the Science Foundation of Ireland under Grant 07/IN.1/I1838. Recommended by Associate Editor P. Shi.

The authors are with the Hamilton Institute, National University of Ireland, Maynooth, Co. Kildare, Maynooth, Ireland (e-mail: zhan.shu@nuim.ie; hustd8@yahoo.cn; hustd8@gmail.com; richard.middleton@nuim.ie).

Color versions of one or more of the figures in this technical note are available online at http://ieeexplore.iee.org.

Digital Object Identifier 10.1109/TAC.2011.2135270 
poles, transmitted power, and channel noise variances for both continuous-time and discrete-time systems. It is revealed that there exists an inherent lower bound on the required transmitted power if an unstable plant needs to be stabilized. We further obtain lower bounds on required channel capacity for stabilization. The lower bound for the continuous-time case can always be achieved by a pair of memoryless LTI encoder and decoder, while for the discrete-time case its achievability via LTI encoders/decoders is not guaranteed generally. The discrepancy between continuous-time and discrete-time systems may be explained by the different power allocations for Shannon limits.

\section{Notation}

Throughout this technical note, $\mathbb{C}^{-}, \mathbb{C}^{+}, \mathbb{D}$, and $\overline{\mathbb{D}}^{c}$ represent the open-left half of the complex plane, open-right half of the complex plane, open unit disk, and complement of the closed unit disk, respectively; $\mathbf{E}\{\cdot\}$ denotes the expectation operator with respect to some probability measure; $\operatorname{tr}(\cdot)$, det $(\cdot)$, spec $(\cdot)$ represent the trace, determinant, and spectrum of a matrix, respectively; A square matrix $A$ is said to be Hurwitz (anti-Hurwitz, respectively) if $\operatorname{spec}(A) \subset \mathbb{C}^{-}\left(\operatorname{spec}(A) \subset \mathbb{C}^{+}\right.$, respectively), and Schur (anti-Schur, respectively) if $\operatorname{spec}(A) \subset \mathbb{D}$ $\left(\operatorname{spec}(A) \subset \overline{\mathbb{D}}^{c}\right.$, respectively); $\lambda_{i}(\cdot)$ and $\rho(\cdot)$ stand for the $i$ th smallest eigenvalue and spectral radius of a matrix, respectively; $\|\cdot\|_{2}$ denotes the $\mathcal{H}_{2}$ norm of an underlying linear system; $(\cdot)_{i}$ represents the $i$ th column of a matrix; $A^{\dagger}$ denotes the unique Moore-Penrose inverse of $A$.

\section{PRELIMINARIES}

The general feedback configuration considered in this technical note is depicted in Fig. 1. The plant, encoder, and decoder are all linear, and have the following realizations:

$$
\begin{aligned}
& \mathcal{G}:\left\{\begin{aligned}
\delta x(t) & =A x(t)+B u(t) \\
y(t) & =x(t)
\end{aligned}\right. \\
& \mathcal{K}_{e}:\left\{\begin{aligned}
\delta x_{e}(t) & =K_{e A} x_{e}(t)+K_{e B} x(t) \\
s(t) & =K_{e C} x_{e}(t)+K_{e D} x(t)
\end{aligned}\right. \\
& \mathcal{K}_{d}:\left\{\begin{aligned}
\delta x_{d}(t) & =K_{d A} x_{d}(t)+K_{d B} r(t) \\
u(t) & =K_{d C} x_{d}(t)+K_{d D} r(t)
\end{aligned}\right.
\end{aligned}
$$

where $x \in \mathbb{R}^{n}, u \in \mathbb{R}^{m}, s \in \mathbb{R}^{m}$, and $r \in \mathbb{R}^{m}$ are the system state, the control input, the channel input, and the channel output, respectively; $\delta$ represents the differential operator $\delta x(t) \triangleq \mathrm{d} x(t) / \mathrm{d} t, t \in \mathbb{R}$ for the continuous-time case, and the shift operator $\delta x(t) \triangleq x(t+1)$, $t \in \mathbb{Z}$ for the discrete-time case, respectively; $K_{e} \triangleq\left[\begin{array}{ll}K_{e A} & K_{e B} \\ K_{e C} & K_{e D}\end{array}\right]$ and $K_{d} \triangleq\left[\begin{array}{ll}K_{d A} & K_{d B} \\ K_{d C} & K_{d D}\end{array}\right]$ are matrices to be determined. The channel input and output have the relation $r(t)=s(t)+n_{\sigma}(t)$, where $n_{\sigma}$ is a Gaussian white noise with power spectral density $\Sigma^{2}$, where $\Sigma=$ $\operatorname{diag}\left(\sigma_{1}, \sigma_{2}, \ldots, \sigma_{m}\right)>0^{1}$. We therefore rewrite the channel equation as

$$
r(t)=s(t)+\Sigma n(t)
$$

where $n$ is a zero mean, circularly symmetric, unit variance additive white Gaussian noise process. Similar to the single input case [10], all signals are required to converge to a stationary distribution, and the channel input has to satisfy the total power constraint

$$
\operatorname{POW}(s) \triangleq \mathbf{E}\left\{\|s(t)\|^{2}\right\}<\mathcal{P}
$$

for some prescribed $\mathcal{P}$. From (4) and the theory of $\mathcal{H}_{2}$ control, $\mathbf{E}\left\{\|s(t)\|^{2}\right\}=\left\|\mathcal{T}_{s n}\right\|_{2}^{2}$, where $\mathcal{T}_{s n}$ represents the resulting

${ }^{1}$ Without loss of generality, any additive white Gaussian noise, with non-degenerate covariance matrix can be transformed to this diagonal case

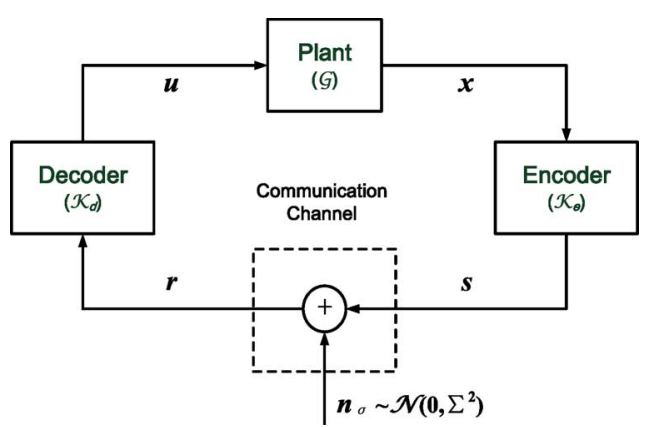

Fig. 1. Feedback configuration over a parallel Gaussian channel.

closed-loop system, and therefore the constraint imposed on the channel input can be reformulated as $\left\|\mathcal{T}_{s n}\right\|_{2}^{2}<\mathcal{P}$.

For technical simplicity, we take the following assumptions on $A$, $B$, and $\Sigma$.

Assumption 1: The system matrix $A \in \mathbb{R}^{n \times n}$ and the input matrix $B \in \mathbb{R}^{n \times m}$ have the structure

$$
A=\operatorname{diag}\left(A_{u}, A_{s}\right), B=\left[\begin{array}{ll}
B_{u}^{T} & B_{s}^{T}
\end{array}\right]^{T}
$$

where $A_{s} \in \mathbb{R}^{n_{s} \times n_{s}}$ is Hurwitz (continuous-time systems) or Schur (discrete-time systems), and $A_{u} \in \mathbb{R}^{n_{u} \times n_{u}}$ is anti-Hurwitz (continuous-time systems) or anti-Schur (discrete-time systems). Note that $A$ has no eigenvalues on the stability boundary ${ }^{2}$.

Assumption 2: $\Sigma$ satisfies

$$
\sigma_{1} \leq \sigma_{2} \leq \cdots \leq \sigma_{m}
$$

Assumption 3: $\left(A_{u}, B_{u}\right)$ is controllable (continuous-time) or reachable (discrete-time).

Note that if $A$ has no eigenvalues on the stability boundary, one can always use appropriate coordination transformation and renumber the control input such that (6) and (7) hold. The controllability or reachability of $\left(A_{u}, B_{u}\right)$ is essential for the stabilization problem considered in this technical note. We end this section by giving a lemma which will be used frequently in the sequel.

Lemma 1 ([15]): For any positive semidefinite matrices $M, N \in \mathbb{R}^{n \times n}, \sum_{i=1}^{n} \lambda_{i}(M) \lambda_{n-i+1}(N) \leq \operatorname{tr}(M N) \leq$ $\sum_{i=1}^{n} \lambda_{i}(M) \lambda_{i}(N)$.

\section{Continuous-Time CASE}

\section{A. Single Encoder and Inequalities for Stabilizability}

In this subsection, we consider the case without decoder, i.e., $K_{d}=$ $I$, and derive necessary/sufficient conditions for stabilizability. Direct manipulations give the closed-loop system

$$
\mathcal{T}_{s n}: \quad \delta x_{c l}(t)=A_{c l} x_{c l}(t)+B_{c l} \Sigma n(t), s(t)=C_{c l} x_{c l}(t)
$$

where $x_{c l}(t)=\left[\begin{array}{ll}x^{T}(t) & x_{e}^{T}(t)\end{array}\right]^{T}$ and

$$
\begin{aligned}
A_{c l} & =\left[\begin{array}{cc}
A+B K_{e A} & B K_{e C} \\
K_{e B} & K_{e A}
\end{array}\right], B_{c l}=\left[\begin{array}{l}
B \\
0
\end{array}\right], \\
C_{c l} & =\left[\begin{array}{ll}
K_{e D} & K_{e C}
\end{array}\right] .
\end{aligned}
$$

Thus, the $\mathcal{H}_{2}$ norm from $n$ to $s$, provided that $A_{c l}$ is Hurwitz, can be evaluated as $\left\|\mathcal{T}_{s n}\right\|_{2}^{2}=\operatorname{tr}\left(\Sigma B_{c l}^{T} Q B_{c l} \Sigma\right)$, where $Q$ satisfies

$$
Q A_{c l}+A_{c l}^{T} Q+C_{c l}^{T} C_{c l}=0 .
$$

2"Stability boundary" stands for the imaginary axis for continuous-time systems and the unit circle for discrete-time systems. 
The following theorem establishes a relationship between stabilizability and transmitted power.

Theorem 1: The system in (1), subject to Assumptions 1-3 and power constraint (5), is stabilizable via an encoder in (2) only if

$$
2 \sum_{i=1}^{n_{u}} \operatorname{Re}\left\{p_{i}\right\}<\frac{\mathcal{P}}{\sigma_{1}^{2}}
$$

where $\operatorname{spec}\left(A_{u}\right)=\left\{p_{i}: i=1,2, \ldots, n_{u}\right\}$. Conversely, suppose that

$$
2 \sum_{i=1}^{n_{u}} \operatorname{Re}\left\{p_{i}\right\}<\frac{\mathcal{P}}{\sigma_{m}^{2}}
$$

then the system in (1) is stabilizable, and the static encoder $K_{e}=$ - $\left[\begin{array}{ll}B_{u}^{T} P_{u} & 0\end{array}\right]$ is optimal ${ }^{3}$.

Proof: First, lower and upper bounds on the infimum of $\left\|\mathcal{T}_{s n}\right\|_{2}^{2}$ achieved by a stabilizing encoder will be derived. Since the Hamiltonian matrix $\left[\begin{array}{cc}A_{u} & -B_{u} B_{u}^{T} \\ 0 & -A_{u}^{T}\end{array}\right]$ has no eigenvalues on the $j \omega$-axis, and $\left(A_{u},-B_{u} B_{u}^{T}\right)$ is controllable (by the controllability of $\left(A_{u}, B_{u}\right)$ ), the Riccati equation $P_{u} A_{u}+A_{u}^{T} P_{u}-P_{u} B_{u} B_{u}^{T} P_{u}=0$ has a unique symmetric solution $P_{u}$ such that $A_{u}-B_{u} B_{u}^{T} P_{u}$ is Hurwitz. The fact that $\left(0, A_{u}\right)$ has no stable unobservable mode further implies that $P_{u}>0$ [16, Theorem 13.7]. Now, define $\bar{P} \triangleq \operatorname{diag}\left(P_{u}, 0\right)$, where the partition is compatible with (6). It is easy to show that

$$
\bar{P} A+A^{T} \bar{P}^{T}-\bar{P} B B^{T} \bar{P}=0 .
$$

Furthermore, define $P \triangleq \operatorname{diag}(\bar{P}, 0)$, where the partition is compatible with (9). Then, simple algebraic manipulations together with (10) and (13) give that

$$
(Q-P) A_{c l}+A_{c l}^{T}(Q-P)+\left(C_{c l}+B_{c l}^{T} P\right)^{T}\left(C_{c l}+B_{c l}^{T} P\right)=0 .
$$

Since $A_{c l}$ is Hurwitz, one has that $Q-P \geq 0$, which implies that $\left\|\mathcal{T}_{s n}\right\|_{2}^{2} \geq \operatorname{tr}\left(\Sigma B_{c l}^{T} P B_{c l} \Sigma\right)$. The equality can be achieved by setting $C_{c l}=-B_{c l}^{T} P$, for which the corresponding encoder becomes $s(t)=$ - $\left[\begin{array}{ll}B_{u}^{T} P_{u} & 0\end{array}\right] x(t)$, and $A_{c l}$ remains Hurwitz due to the stability of $A_{u}-B_{u} B_{u}^{T} P_{u}$. With this relationship, one has that

$$
\begin{aligned}
\inf _{\mathcal{K}_{e} \text { is stabilizing }}\left\|\mathcal{T}_{s n}\right\|_{2}^{2} & =\operatorname{tr}\left(\Sigma B_{c l}^{T} P B_{c l} \Sigma\right) \\
& =\operatorname{tr}\left(\Sigma B_{u}^{T} P_{u} B_{u} \Sigma\right) .
\end{aligned}
$$

Noting that $P_{u}$ satisfies the Riccati equation mentioned above, one obtains

$$
\begin{aligned}
\operatorname{tr}\left(B_{u}^{T} P_{u} B_{u}\right) & =\operatorname{tr}\left(P_{u}^{1 / 2} B_{u} B_{u}^{T} P_{u}^{1 / 2}\right) \\
& =2 \operatorname{tr}\left(P_{u}^{-1 / 2} A_{u}^{T} P_{u}^{1 / 2}\right)=2 \sum_{i=1}^{n_{u}} \operatorname{Re}\left\{p_{i}\right\} .
\end{aligned}
$$

Therefore, applying Lemma 1 to matrix $\left(B_{u}^{T} P_{u} B_{u}\right) \Sigma^{2}$ yields that

$$
2 \sigma_{1}^{2} \sum_{i=1}^{n_{u}} \operatorname{Re}\left\{p_{i}\right\} \leq \inf _{\mathcal{K}_{e} \text { is stabilizing }}\left\|\mathcal{T}_{s n}\right\|_{2}^{2} \leq 2 \sigma_{m}^{2} \sum_{i=1}^{n_{u}} \operatorname{Re}\left\{p_{i}\right\} .
$$

If the system in (1) is stabilizable, then from this inequality it follows that (11) holds. If (12) holds, then, from this inequality, there must exist an encoder such that the closed-loop system is stable and POW $(s)<$ $\mathcal{P}$.

Corollary 1: If the system in (1), subject to Assumptions 1-3 and power constraint (5), is stabilizable for $\Sigma=\epsilon I$, it is stabilizable for any $\Sigma \leq \epsilon I$ as well. If the system is not stabilizable for $\Sigma=\epsilon I$, then it is not stabilizable for any $\Sigma \geq \epsilon I$ as well.

\footnotetext{
${ }^{3}$ The "optimality" considered here means that the required transmitted power is minimal.
}

Proof: Stabilizability for $\Sigma=\epsilon I$ is equivalent to $2 \epsilon^{2} \sum_{i=1}^{n_{u}} \operatorname{Re}\left\{p_{i}\right\}=\inf _{\mathcal{K}_{e} \text { is stabilizing }}\left\|\mathcal{T}_{s n}\right\|_{2}^{2}<\mathcal{P}$. This implies that, for $\Sigma \leq \epsilon I, 2 \sum_{i=1}^{n_{u}} \operatorname{Re}\left\{p_{i}\right\}<\mathcal{P} / \sigma_{m}^{2}$, which by (12) implies the stabilizability. The proof of instabilizability is similar.

The lower bound in (14) is closely related to the Shannon limit of parallel Gaussian channels, as will be clear later, but generally unachievable. It is natural to ask whether it is possible to make the bound always achievable. In the next subsection, we shall give a positive answer to this question, and show that adding a static decoder after receiving signals from the channels is sufficient for this.

\section{B. Achievability of Optimal Transmitted Power via Encoder Plus Decoder}

We first restrict attention to static decoders. Generally speaking, simultaneous design of $K_{e}$ and $K_{d}$ may be complex, since it is equivalent to an $\mathcal{H}_{2}$ problem subject to a structural constraint. However, the weak separation property that the optimal encoder is uniquely determined if a decoder is given enables one to split the design into two steps: $K_{d}$ first and $K_{e}$ later. In the remaining part of this subsection, two achievability lemmas will be given first, and then a theorem showing that the lower bound in (14) is always achievable will be established. Before proceeding, define

$$
\boldsymbol{M}_{d} \triangleq\left\{k \mid \sigma_{k}^{2}=\sigma_{1}^{2}, k=1,2, \ldots, m\right\} .
$$

Lemma 2 (Sufficient Condition for Achievability): If there exists a $K_{d}$ such that $\left(B_{u} K_{d}\right)_{i}=0$ for $i \notin \boldsymbol{M}_{d}$, and $\left(A_{u}, B_{u} K_{d}\right)$ is controllable, then the lower bound in (14) is achievable.

Proof: The controllability of $\left(A_{u}, B_{u} K_{d}\right)$ ensures the existence of the optimal encoder $s(t)=-\left[\begin{array}{ll}B_{u}^{T} P_{u} & 0\end{array}\right] x(t)$, and the corresponding transmitted power is $\operatorname{tr}\left(K_{d}^{T} B_{u}^{T} P_{u} B_{u} K_{d} \Sigma^{2}\right)$. Noting that the $i$ th diagonal position, $i \notin M_{d}$, of $K_{d}^{T} B_{u}^{T} P_{u} B_{u} K_{d}$ is zero, one has that

$$
\begin{aligned}
\operatorname{tr}\left(K_{d}^{T} B_{u}^{T} P_{u} B_{u} K_{d} \Sigma^{2}\right) & =\sigma_{1}^{2} \operatorname{tr}\left(K_{d}^{T} B_{u}^{T} P_{u} B_{u} K_{d}\right) \\
& =2 \sigma_{1}^{2} \sum_{i=1}^{n_{u}} \operatorname{Re}\left\{p_{i}\right\} .
\end{aligned}
$$

Lemma 3 (Necessary Condition for Achievability): If the lower bound in (14) is achievable by some $K_{d}$ and corresponding $K_{e}$, then $\left(A_{u}, B_{u} K_{d}\right)$ is controllable and $\left(B_{u} K_{d}\right)_{i}=0$ for $i \notin \boldsymbol{M}_{d}$.

Proof: Represent $K_{d}^{T} B_{u}^{T} P_{u} B_{u} K_{d}$ as

$$
K_{d}^{T} B_{u}^{T} P_{u} B_{u} K_{d}=\left[\begin{array}{cccc}
z_{1} & * & \cdots & * \\
* & z_{2} & \cdots & * \\
\vdots & \vdots & \ddots & * \\
* & * & * & z_{m}
\end{array}\right] .
$$

Obviously, $z_{j} \geq 0, j=1,2, \ldots, m$, and $\operatorname{tr}\left(\Sigma K_{d}^{T} B_{u}^{T} P_{u} B_{u} K_{d} \Sigma\right)=$ $\sum_{i=1}^{m} z_{i} \sigma_{i}^{2}$. If there exists $i \notin \boldsymbol{M}_{d} \neq\{1,2, \ldots, m\}$ such that $z_{i} \neq 0$, then $\Sigma \neq \sigma I$ for some $\sigma$, and thus $\sum_{i=1}^{m} z_{i} \sigma_{i}^{2}>\sigma_{1}^{2} \sum_{i=1}^{m} z_{i}=$ $2 \sigma_{1}^{2} \sum_{i=1}^{n_{u}} \operatorname{Re}\left\{p_{i}\right\}$, which contradicts with the fact that $\sum_{i=1}^{m} z_{i} \sigma_{i}^{2}=$ $2 \sigma_{1}^{2} \sum_{i=1}^{n_{u}} \operatorname{Re}\left\{p_{i}\right\}$. Hence $z_{i}=0$ for $i \notin \boldsymbol{M}_{d}$. On the other hand, $z_{i}=\left(B_{u} K_{d}\right)_{i}^{T} P_{u}\left(B_{u} K_{d}\right)_{i}$. Therefore, $\left(B_{u} K_{d}\right)_{i}=0$ for $i \notin M_{d}$ due to $P_{u}>0$. If $M_{d}=\{1,2, \ldots, m\}$, then $\Sigma=\sigma I$ for some $\sigma$, and there is no $i \notin M_{d}$. The result is obvious. In addition, the stability of $A_{u}+B_{u} K_{d} K_{e}$ implies that $\left(A_{u}, B_{u} K_{d}\right)$ is stabilizable, and thus $\left(A_{u}, B_{u} K_{d}\right)$ is controllable due to $\operatorname{Re}\left\{p_{i}\right\}>0$.

These two achievability lemmas establish a necessary and sufficient condition for stabilizability with optimal transmitted power, and show that optimal stabilization can be achieved only when all subchannels with noise variance strictly larger than $\sigma_{1}^{2}$ are abandoned. As will be shown later, this transmission scheme also maximizes the mutual information between channel input and output, and therefore achieves the Shannon limit of (continuous-time) parallel Gaussian channels. 
Theorem 2: For the system in (1), subject to Assumptions 1-3 and power constraint (5), the lower bound in (14) is always achievable by some static $K_{d}$ and $K_{e}$.

Proof: Since $\left(A_{u}, B_{u}\right)$ is controllable, for any left eigenvector $v_{i}$ of $A_{u}$ such that $v_{i}^{*} A_{u}=v_{i}^{*} p_{i}, i=1,2, \ldots, l, v_{i}^{*} B_{u} \neq 0$. Thus, it suffices to prove that there exists a $K_{d}$ such that $v_{i}^{*} B_{u} K_{d} \neq 0$ for any left eigenvector $v_{i}$. Choose an arbitrary nonzero vector $h_{0}$, and write $\left[\begin{array}{llll}v_{1} & v_{2} & \cdots & v_{l}\end{array}\right]^{*} B_{u} h_{0}=\left[\begin{array}{llll}w_{1} & w_{2} & \cdots & w_{l}\end{array}\right]^{*}$. If $w_{i}=0$ for some $i$, then perturb $h_{0}$ to $h_{1}$ such that $w_{i} \neq 0$ and other nonzero $w_{i}$ remains nonzero. This is always possible, since $v_{i}^{*} B_{u} \neq 0$. Repeat this process until an $h_{q}$ is obtained such that all $w_{i}$ are nonzero. Construct $K_{d}$ such that $\left(K_{d}\right)_{i}=0, i \notin \boldsymbol{M}_{d}$, and $\left(K_{d}\right)_{i}=h_{q}, i \in \boldsymbol{M}_{d}$. From the property of $h_{q}$, it follows that $v_{i}^{*} B_{u} K_{d} \neq 0$, and thus $\left(A_{u}, B_{u} K_{d}\right)$ is controllable. Applying Lemma 2 yields the desired result.

It is noted that the lower bound in (14) is solely related to the poles in $\mathbb{C}^{+}$and the minimal noise variance. If one connects a decoder in (3) to a plant in (1), one could obtain an equivalent plant with the realization $\left(\left[\begin{array}{cc}A & B K_{d C} \\ 0 & K_{d A}\end{array}\right],\left[\begin{array}{c}B K_{d D} \\ K_{d B}\end{array}\right], I\right)$. The fact that $A_{n e w}$ includes all unstable poles of $A$ together with Theorem 2 indicates that dynamic decoders are unnecessary for the stabilization problem considered here.

\section{Channel Capacity and Stabilizability}

The information capacity of the power constrained parallel Gaussian channel [17] is

$$
\mathcal{C} \triangleq \max _{\operatorname{POW}(s) \leq \mathcal{P}} I(s ; r)=\max _{\operatorname{POW}(s) \leq \mathcal{P}} \sum_{i=1}^{m} \frac{\mathcal{P}_{i}}{2 \sigma_{i}^{2}} \log _{2} \mathrm{e}
$$

where $I(s ; r)$ is the mutual information of $s$ and $r, \mathcal{P}_{i} \geq 0$ is transmitted power of the $i$ th subchannel, and $\sum_{i=1}^{m} \mathcal{P}_{i} \leq \mathcal{P}$. Noting that $\sum_{i=1}^{m}\left(\mathcal{P}_{i} / 2 \sigma_{i}^{2}\right) \log _{2} \mathrm{e} \leq \sum_{i=1}^{m}\left(\mathcal{P}_{i} / 2 \sigma_{1}^{2}\right) \log _{2} \mathrm{e} \leq\left(\mathcal{P} / 2 \sigma_{1}^{2}\right) \log _{2} \mathrm{e}$ where the first equality is achieved when $\mathcal{P}_{i}=0, i \notin \boldsymbol{M}_{d}$, one has that

$$
\mathcal{C}=\frac{\mathcal{P}}{2 \sigma_{1}^{2}} \log _{2} \mathrm{e} \quad \frac{\text { bits }}{\text { second }} .
$$

This together with Theorems 1 and 2 yields the following result.

Theorem 3: The system in (1), subject to Assumptions 1-3 and power constraint (5), is stabilizable via a pair of encoder $K_{e}$ and decoder $K_{d}$ if and only if the channel capacity satisfies

$$
\mathcal{C}>\left(\sum_{i=1}^{n_{u}} \operatorname{Re}\left\{p_{i}\right\}\right) \log _{2} \mathrm{e}
$$

All the results mentioned above manifest clearly that memoryless LTI encoders/decoders are sufficient for stabilizing a continuous-time linear systems while achieving the lower bound on required channel capacity. The proof of Theorem 2 has suggested an approach to finding a desired $K_{d}$, and, once $K_{d}$ is fixed, the optimal $K_{e}$ can be obtained readily by solving corresponding Riccati equation. It should be emphasized here that, unlike the single-input case, both $K_{e}$ and $K_{d}$ in general must be utilized in order to achieve the optimal transmitted power.

\section{DisCRETE-TIME CASE}

\section{A. Single Encoder and Inequalities for Stabilizability}

Similar to the continuous-time case, the closed-loop system with a single encoder is described by (8), and the $\mathcal{H}_{2}$ norm from $n$ to $s$, provided that $A_{c l}$ is Schur, can be evaluated as $\left\|\mathcal{T}_{s n}\right\|_{2}^{2}=\operatorname{tr}\left(\Sigma B_{c l}^{T} Q B_{c l} \Sigma\right)$, where $Q$ satisfies $A_{c l}^{T} Q A_{c l}-$ $Q+C_{c l}^{T} C_{c l}=0$. Before presenting the main result of this subsection, define $f(s) \triangleq \sqrt[s]{\prod_{i=1}^{s} \sigma_{i}^{2} \prod_{k=1}^{n u}\left|\phi_{k}\right|^{2}}$, where
$\operatorname{spec}\left(A_{u}\right)=\left\{\phi_{k}: k=1,2, \ldots, n_{u}\right\}$, and $1 \leq s \leq m$ is a natural number.

Lemma 4: Regarding $f(s)$, one has that

a) If $\sigma_{r}^{2}<f(r)$ for some $r \in \mathbb{N}$, then, for any $s \in \mathbb{N}, s<r$ : $\sigma_{s}^{2}<f(s)$.

b) $f(s) \leq \sigma_{1}^{2} \prod_{k=1}^{n_{u}}\left|\phi_{k}\right|^{2}, \forall s \in \mathbb{N}$ such that $\sigma_{s}^{2}<f(s)$. Proof:

a) $\sigma_{r}^{2}<f(r)$ implies that $\left(\sigma_{r}^{2}\right)^{r}<\prod_{i=1}^{r} \sigma_{i}^{2} \prod_{k=1}^{n_{u}}\left|\phi_{k}\right|^{2}$, and thus $\left(\sigma_{r-1}^{2}\right)^{r-1} \leq\left(\sigma_{r}^{2}\right)^{r-1}<\prod_{i=1}^{r-1} \sigma_{i}^{2} \prod_{k=1}^{n_{u}}\left|\phi_{k}\right|^{2}$, which means that $\sigma_{r-1}^{2}<f(r-1)$. Repeating this argument gives the result.

b) According to a), there must exist an $r$ such that $\sigma_{s}^{2}<f(s)$, $\forall s \leq r$, and $\sigma_{s}^{2} \geq f(s), \forall s>r$. From this observation, it suffices to prove b) for $1 \leq s \leq r$. When $s=1$, this is obvious. Assume that this holds for $s=l$, i.e., $f(l) \leq \sigma_{1}^{2} \prod_{k=1}^{n_{u}}\left|\phi_{k}\right|^{2}$. Then, for $s=l+1 \leq r$

$$
\begin{aligned}
f(l+1) & =\sqrt[l+1]{f^{l}(l) \sqrt[l+1]{\sigma_{l+1}^{2}}} \\
& \leq \sqrt[l+1]{\left(\sigma_{1}^{2} \prod_{k=1}^{n_{u}}\left|\phi_{k}\right|^{2}\right)^{l}} \sqrt[l+1]{\sigma_{l+1}^{2}} \\
& <\sqrt[l+1]{\left(\sigma_{1}^{2} \prod_{k=1}^{n_{u}}\left|\phi_{k}\right|^{2}\right)^{l}} \sqrt[l+1]{f(l+1)}
\end{aligned}
$$

where the second inequality is due to a) and $l+1 \leq r$. Multiplying both sides of the above inequality by $f(l+1)^{-1 /(l+1)}$ yields that $\mathrm{b}$ ) holds for $s=l+1$. The result follows by induction.

Theorem 4: The system in (1), subject to Assumptions 1-3 and power constraint (5), is stabilizable via an encoder in (2) only if

$$
\sqrt[v]{\prod_{k=1}^{n_{u}}\left|\phi_{k}\right|^{2}}<\frac{\mathcal{P}+\sum_{j=1}^{v} \sigma_{j}^{2}}{v \sqrt[v]{\prod_{i=1}^{v} \sigma_{i}^{2}}}
$$

where $v \leq m$ is the largest integer such that $\sigma_{v}^{2}<f(v)$. Conversely, suppose that

$$
\prod_{k=1}^{n_{u}}\left|\phi_{k}\right|^{2}<\frac{\mathcal{P}}{\sigma_{m}^{2}}+1
$$

then the system in (1) is stabilizable, and the static encoder $K_{e}=$ $-\left[\left(I+B_{u}^{T} P_{u} B_{u}\right)^{-1} \quad B_{u}^{T} P_{u} A_{u} \quad 0\right]$ is optimal.

Proof: Since the Simplectic matrix $\left[\begin{array}{cc}A_{u} & -B_{u} B_{u}^{T}\left(A_{u}^{T}\right)^{-1} \\ 0 & \left(A_{u}^{T}\right)^{-1}\end{array}\right]$ has no eigenvalues on the unit circle, and $\left(A_{u},-B_{u} B_{u}^{T}\right)$ is reachable (by the reachability of $\left(A_{u}, B_{u}\right)$ ), the Riccati equation $A_{u}^{T} P_{u} A_{u}-$ $P_{u}-A_{u}^{T} P_{u} B_{u}\left(I+B_{u}^{T} P_{u} B_{u}\right)^{-1} B_{u}^{T} P_{u} A_{u}=0$ has a unique symmetric solution such that $A_{u}-B_{u}\left(I+B_{u}^{T} P_{u} B_{u}\right)^{-1} B_{u}^{T} P_{u} A_{u}$ is Schur. In addition, the fact that $\left(0, A_{u}\right)$ has no stable unobservable mode implies that $P_{u}>0$ [16, Theorem 21.11]. Now, define $\bar{P} \triangleq \operatorname{diag}\left(P_{u}, 0\right)$ and $P \triangleq \operatorname{diag}(\bar{P}, 0)$, where the partition is compatible with (6) and (9), respectively. By following a similar line as used in the proof of Theorem 1, one obtains that

$$
\begin{aligned}
A_{c l}^{T}(Q-P) A_{c l}- & (Q-P) \\
& +\left(C_{c l}+F\right)^{T}\left(I+B_{c l}^{T} P B_{c l}\right)\left(C_{c l}+F\right)=0
\end{aligned}
$$

where $F=\left[\left(I+B^{T} \bar{P} B\right)^{-1} \quad B^{T} \bar{P} A \quad 0\right]$. Since $A_{c l}$ is Schur, one obtains that $Q-P \geq 0$, which results in $\left\|\mathcal{T}_{s n}\right\|_{2}^{2} \geq \operatorname{tr}\left(\Sigma B_{c l}^{T} P B_{c l} \Sigma\right)$. The equality can be achieved by setting $K_{e D}=-\left[\left(I+B_{u}^{T} P_{u} B_{u}\right)^{-1} B_{u}^{T} P_{u} A_{u} \quad 0\right]$, $K_{e C}=0$, for which $A_{c l}$ remains Schur due to the stability of $A_{u}-B_{u}\left(I+B_{u}^{T} P_{u} B_{u}\right)^{-1} B_{u}^{T} P_{u} A_{u}$. Noting that $P_{u}$ satisfies the Riccati equation mentioned above, the spectrum of the closed-loop 
system can be decomposed as

$$
\begin{aligned}
& \operatorname{spec}\left(A-B\left(I+B^{T} \bar{P} B\right)^{-1} B^{T} \bar{P} A\right) \\
& =\operatorname{spec}\left(P_{u}^{-1} A_{u}^{-T} P_{u}\right) \cup \operatorname{spec}\left(A_{s}\right) \\
& =\operatorname{spec}\left(A_{u}^{-1}\right) \cup \operatorname{spec}\left(A_{s}\right) .
\end{aligned}
$$

According to the Matrix Inverse Lemma [18], $A$ $B\left(I+B^{T} \bar{P} B\right)^{-1} B^{T} \bar{P} A=\left(I+B B^{T} \bar{P}\right)^{-1} A$. This together with (18) implies that

$\operatorname{det}\left(\left(I+B_{u}^{T} P_{u} B_{u}\right)^{-1}\right) \operatorname{det}\left(A_{u}\right) \operatorname{det}\left(A_{s}\right)$ $=\operatorname{det}\left(A_{u}^{-1}\right) \operatorname{det}\left(A_{s}\right)$

and thus $\operatorname{det}\left(I+B_{u}^{T} P_{u} B_{u}\right)=\prod_{k=1}^{n_{u}}\left|\phi_{k}\right|^{2}$. With this and Lemma 1, one obtains that

$$
\begin{aligned}
\sum_{i=1}^{m} \sigma_{i}^{2} \lambda_{m-i+1}\left(B_{u}^{T} P_{u} B_{u}\right) & \leq \inf _{\mathcal{K}_{e} \text { is stabilizing }}\left\|\mathcal{T}_{s n}\right\|_{2}^{2} \\
& \leq \sum_{i=1}^{m} \sigma_{i}^{2} \lambda_{i}\left(B_{u}^{T} P_{u} B_{u}\right)
\end{aligned}
$$

where $\lambda_{i}\left(B_{u}^{T} P_{u} B_{u}\right) \geq 0$ and $\prod_{i=1}^{m}\left(1+\lambda_{i}\left(B_{u}^{T} P_{u} B_{u}\right)\right)=$ $\prod_{k=1}^{n_{u}}\left|\phi_{k}\right|^{2}$. Let us now evaluate both sides of (19).

1) By using the arithmetic and geometric mean inequality, one has that

$$
\begin{aligned}
& \sum_{i=1}^{m} \sigma_{i}^{2} \lambda_{m-i+1}\left(B_{u}^{T} P_{u} B_{u}\right) \\
& \left.\geq m \sqrt[m]{\prod_{i=1}^{m}\left\{\sigma_{i}^{2}\left[\lambda_{m-i+1}\left(B_{u}^{T} P_{u} B_{u}\right)+1\right]\right.}\right\}-\sum_{i=1}^{m} \sigma_{i}^{2} \\
& =m f(m)-\sum_{i=1}^{m} \sigma_{i}^{2}
\end{aligned}
$$

where the equality is achieved when

$$
\begin{aligned}
& \sigma_{1}^{2}\left(1+\lambda_{m}\left(B_{u}^{T} P_{u} B_{u}\right)\right) \\
& =\sigma_{2}^{2}\left(1+\lambda_{m-1}\left(B_{u}^{T} P_{u} B_{u}\right)\right) \\
& =\ldots=\sigma_{m}^{2}\left(1+\lambda_{1}\left(B_{u}^{T} P_{u} B_{u}\right)\right)=f(m) .
\end{aligned}
$$

However, since $\lambda_{i}\left(B_{u}^{T} P_{u} B_{u}\right)$ cannot be negative, the lower bound may not be achieved if $\sigma_{i}^{2}$ is too large. More specifically, if $\sigma_{m}^{2} \geq f(m)$, then the optimal $\lambda_{1}\left(B_{u}^{T} P_{u} B_{u}\right)$ is 0 . Repeat this process until $\sigma_{v}^{2}<f(v)$ for some $v$. Then, the possibly achieved bound is

$$
\sum_{i=1}^{m} \sigma_{i}^{2} \lambda_{m-i+1}\left(B_{u}^{T} P_{u} B_{u}\right) \geq v f(v)-\sum_{i=1}^{v} \sigma_{i}^{2} .
$$

One may worry about that whether $\lambda_{i}\left(B_{u}^{T} P_{u} B_{u}\right)$ would have to exceed its maximum $\prod_{k=1}^{n_{u}}\left|\phi_{k}\right|^{2}-1$ if $\sigma_{i}^{2}$ is very small. Applying Lemma 4 yields that $\forall i \leq v, \sigma_{i}^{2}<f(i)$ and $f(i) / \sigma_{i}^{2} \leq$ $\sigma_{1}^{2} \prod_{k=1}^{n_{u}}\left|\phi_{k}\right|^{2} / \sigma_{i}^{2}=\prod_{k=1}^{n_{u}}\left|\phi_{k}\right|^{2}$, and thus this situation would never occur.

2) It can be shown by using the Lagrangian multiplier method that $\sum_{i=1}^{n_{u}} \sigma_{i}^{2} \lambda_{i}\left(B_{u}^{T} P_{u} B_{u}\right)$ has only one minimum point, and thus $\sum_{i=1}^{n_{u}} \sigma_{i}^{2} \lambda_{i}\left(B_{u}^{T} P_{u} B_{u}\right)$ takes its maximum at boundary points. Simple calculations give that

$$
\sum_{i=1}^{m} \sigma_{i}^{2} \lambda_{i}\left(B_{u}^{T} P_{u} B_{u}\right) \leq \sigma_{m}^{2}\left(\prod_{k=1}^{n_{u}}\left|\phi_{k}\right|^{2}-1\right)
$$

where the equality holds when $\lambda_{1}=\lambda_{2}=\cdots=\lambda_{m-1}=0$. The results follows immediately from the two bounds.
Corollary 2: If the system in (1), subject to Assumptions 1-3 and power constraint (5), is stabilizable for $\Sigma=\epsilon I$, it is stabilizable for any $\Sigma \leq \epsilon I$ as well. If the system is not stabilizable for $\Sigma=\epsilon I$, then it is not stabilizable for any $\Sigma \geq \epsilon I$ as well.

Proof: Stabilizability for $\Sigma=\epsilon I$ is equivalent to $\epsilon^{2} \sum_{i=1}^{m} \lambda_{i}\left(B_{u}^{T} P_{u} B_{u}\right)=\inf _{\mathcal{K}_{e} \text { is stabilizing }}\left\|\mathcal{T}_{s n}\right\|_{2}^{2}<\mathcal{P}$. This implies that, for $\Sigma \leq \epsilon I$

$$
\begin{aligned}
\inf _{\mathcal{K}_{\text {is }} \text { stabilizing }}\left\|\mathcal{T}_{s n}\right\|_{2}^{2} & =\operatorname{tr}\left(\Sigma B_{u}^{T} P_{u} B_{u} \Sigma\right) \\
& \leq \epsilon^{2} \sum_{i=1}^{m} \lambda_{i}\left(B_{u}^{T} P_{u} B_{u}\right)<\mathcal{P}
\end{aligned}
$$

which implies the stabilizability. The proof of instabilizability is similar.

The lower bound in (20), similar to the continuous-time case, is closely associated with Shannon limit, but is generally unachievable by a static encoder. One may speculate from the results for continuous-time systems that the bound can always be achieved by adding a decoder. Unfortunately, this conjecture is not true, as will be revealed in the next subsection.

\section{B. Achievability of Optimal Transmitted Power via Encoder Plus Decoder}

We first restrict attention to static decoders. Before proceeding, define

$$
\mu_{i}^{*}= \begin{cases}\frac{f(v)}{\sigma_{i}^{2}}-1 & i \leq v \\ 0 & i>v\end{cases}
$$

where $v$ is defined as in Theorem 4. In fact, $\mu_{i}^{*}$ is the optimal signal-tonoise ratio for the $i$ th subchannel when the lower bound on channel capacity is achieved. This will be clear along with the development. In the subsequent derivations, unless stated particularly, $P_{u}$ denotes the solution of

$$
\begin{aligned}
A_{u}^{T} P_{u} A_{u}-P_{u}- & A_{u}^{T} P_{u} B_{u} K_{d} \\
& \times\left(I+K_{d}^{T} B_{u}^{T} P_{u} B_{u} K_{d}\right)^{-1} K_{d}^{T} B_{u}^{T} P_{u} A_{u}=0 .
\end{aligned}
$$

Lemma 5 (Sufficient Condition for Achievability): If there exists a $K_{d}$ such that $\left(A_{u}, B_{u} K_{d}\right)$ is reachable and

$$
\left(B_{u} K_{d}\right)_{i}^{T} P_{u}\left(B_{u} K_{d}\right)_{i}=\mu_{i}^{*}
$$

then the lower bound in (20) is achievable.

Proof: The reachability of $\left(A_{u}, B_{u} K_{d}\right)$ ensures the existence of optimal encoder, and thus the corresponding transmitted power is $\operatorname{tr}\left(K_{d}^{T} B_{u}^{T} P_{u} B_{u} K_{d} \Sigma^{2}\right)=\sum_{i=1}^{m} \sigma_{i}^{2} \mu_{i}^{*}$. The proof is completed by employing (22).

Lemma 6 (Necessary Condition for Achievability): If the lower bound in (20) is achievable by some $K_{d}$ and corresponding $K_{e}$, then $\left(A_{u}, B_{u} K_{d}\right)$ is reachable and $K_{d}^{T} B_{u}^{T} P_{u} B_{u} K_{d}=\operatorname{diag}\left\{\mu_{i}^{*}\right\}$.

Proof: The reachability of $\left(A_{u}, B_{u} K_{d}\right)$ follows from the stability of the closed-loop system. Write $K_{d}^{T} B_{u}^{T} P_{u} B_{u} K_{d}$ as (15). Obviously, $z_{j} \geq 0, j=1,2, \ldots, m$, and

$$
\begin{aligned}
\operatorname{tr}\left(\Sigma K_{d}^{T} B_{u}^{T} P_{u} B_{u} K_{d} \Sigma\right) & =\sum_{i=1}^{m} z_{i} \sigma_{i}^{2} \\
& =v \sqrt[v]{\prod_{i=1}^{v} \sigma_{i}^{2} \prod_{k=1}^{n_{u}}\left|\phi_{k}\right|^{2}}-\sum_{i=1}^{v} \sigma_{i}^{2} .
\end{aligned}
$$

From this

$$
v f(v)+\sum_{i=v+1}^{m} \sigma_{i}^{2}=\sum_{i=1}^{m}\left(z_{i}+1\right) \sigma_{i}^{2} .
$$


Let us now evaluate the minimum of the right hand side of (23)

$$
\begin{aligned}
\sum_{i=1}^{m}\left(z_{i}+1\right) \sigma_{i}^{2} & \geq m \sqrt[m]{\prod_{i=1}^{m} \sigma_{i}^{2}\left(z_{i}+1\right)} \\
& \geq m \sqrt[m]{\prod_{i=1}^{m} \sigma_{i}^{2} \operatorname{det}\left(I+K_{d}^{T} B_{u}^{T} P_{u} B_{u} K_{d}\right)} \\
& =m f(m)
\end{aligned}
$$

where the first and the second inequalities come from the arithmetic and geometric means inequality and Hadamard's inequality, respectively. The equality holds if and only if $K_{d}^{T} B_{u}^{T} P_{u} B_{u} K_{d}$ is diagonal (Hadamard's inequality) and $\left(z_{1}+1\right) \sigma_{1}^{2}=\left(z_{2}+1\right) \sigma_{2}^{2}=\cdots=$ $\left(z_{m}+1\right) \sigma_{m}^{2}=f(m)$ (arithmetic and geometric means inequality). However, it has been pointed out in the proof of Theorem 4 that $\sigma_{m}^{2} \geq$ $\sqrt[m]{\prod_{i=1}^{m} \sigma_{i}^{2} \prod_{k=1}^{n_{u}}\left|\phi_{k}\right|^{2}}$. Hence, $z_{m}$ cannot achieve its globally optimal value, and the optimal $z_{m}$ is $z_{m}=0$. Repeating this argument gives that $\sum_{i=1}^{m}\left(z_{i}+1\right) \sigma_{i}^{2} \geq v f(v)+\sum_{i=v+1}^{m} \sigma_{i}^{2}$ where the equality holds only if $K_{d}^{T} B_{u}^{T} P_{u} B_{u} K_{d}$ is diagonal and $\left(z_{1}+1\right) \sigma_{1}^{2}=$ $\left(z_{2}+1\right) \sigma_{2}^{2}=\cdots=\left(z_{v}+1\right) \sigma_{v}^{2}=f(v)$. From this and (23), it follows that $z_{i}=f(v) / \sigma_{i}^{2}-1=\mu_{i}^{*}, i=1,2, \ldots, v$ and $z_{i}=0$, $i=v+1, v+2, \ldots, m$. This completes the proof.

The following theorem and its corollary indicate that the lower bound in (20) is not always achievable by LTI encoders/decoders.

Theorem 5: The lower bound in (20) is achievable by some $K_{d}$ and corresponding $K_{e}$ if and only if $n_{u} \geq v$ and there exist unitary matrices $M$ and $N$ such that the following constrained Sylvester equation has a nonsingular solution $Z$ :

$$
\begin{aligned}
A_{u} Z & =Z(M \Lambda N) \\
\left(B_{u} B_{u}^{\dagger}-I\right) Z \bar{\Lambda} & =0
\end{aligned}
$$

where $\Lambda=\left(I+\bar{\Lambda} \bar{\Lambda}^{T}\right)^{1 / 2}$ and $\bar{\Lambda}=\left[\operatorname{diag}\left\{\sqrt{\mu_{i}^{*}}\right\} \quad 0\right]^{T} \quad\left(n_{u} \geq m\right)$ or $\operatorname{diag}\left\{\sqrt{\mu_{i}^{*}}\right\}\left(n_{u}<m\right)$.

Proof: From Lemmas 5, 6, and $0<P_{u} \in \mathbb{R}^{n_{u} \times n_{u}}$, the lower bound in (20) is achievable if and only if $n_{u} \geq v$ and there exists a $P_{u}>0$ such that

$$
\begin{aligned}
& A_{u}^{T} P_{u} A_{u}-P_{u} \\
& \quad-A_{u}^{T} P_{u} B_{u} K_{d}\left(I+K_{d}^{T} B_{u}^{T} P_{u} B_{u} K_{d}\right)^{-1} K_{d}^{T} B_{u}^{T} P_{u} A_{u}=0
\end{aligned}
$$

and $K_{d}^{T} B_{u}^{T} P_{u} B_{u} K_{d}=\operatorname{diag}\left\{\mu_{i}^{*}\right\}$. By letting $X_{u}=P_{u}^{-1}$, and applying the Matrix Inverse Lemma [18], one obtains an equivalent condition that

$$
\begin{aligned}
A_{u} X_{u} A_{u}^{T}-X_{u} & =B_{u} K_{d} K_{d}^{T} B_{u}^{T} \\
K_{d}^{T} B_{u}^{T} X_{u}^{-1} B_{u} K_{d} & =\operatorname{diag}\left\{\mu_{i}^{*}\right\} .
\end{aligned}
$$

Noting that $X_{u}>0$ if and only if there exists a nonsingular matrix $Z$ such that $X_{u}=Z Z^{T}$. With this decomposition, (25) is equivalent to $\left(Z^{-1} A_{u} Z\right)\left(Z^{-1} A_{u} Z\right)^{T}=I+\left(Z^{-1} B_{u} K_{d}\right)\left(Z^{-1} B_{u} K_{d}\right)^{T}$ and $\left(Z^{-1} B_{u} K_{d}\right)^{T}\left(Z^{-1} B_{u} K_{d}\right)=\operatorname{diag}\left\{\mu_{i}^{*}\right\}$, which, by using singular value decomposition, implies that

$$
\begin{aligned}
\left(Z^{-1} A_{u} Z\right)\left(Z^{-1} A_{u} Z\right)^{T} & =\bar{U} \Lambda^{2} \bar{U}^{T} \\
Z^{-1} B_{u} K_{d} & =\bar{U} \bar{\Lambda} \bar{V}
\end{aligned}
$$

for some unitary matrices $\bar{U}$ and $\bar{V}$. Using singular value decomposition again yields that $(26)$ is equivalent to

$$
\begin{aligned}
\bar{U}^{T} Z^{-1} A_{u} Z \bar{U} & =M \Lambda N \\
B_{u}\left(K_{d} \bar{V}^{T}\right) & =(Z \bar{U}) \bar{\Lambda}
\end{aligned}
$$

for some unitary matrices $M$ and $N$. By re-defining $Z$ and $K_{d}$ as $Z \bar{U} \rightarrow Z$ and $K_{d} \bar{V}^{T} \rightarrow K_{d}$, and noting that the equation $B_{u}\left(K_{d} \bar{V}^{T}\right)=(Z \bar{U}) \bar{\Lambda}$ has a solution $K_{d} \bar{V}^{T}$ if and only if $B_{u} B_{u}^{\dagger}(Z \bar{U}) \bar{\Lambda}=(Z \bar{U}) \bar{\Lambda}$ [18], (27) is further equivalent to (24).

Corollary 3: The lower bound in (20) is achievable only if

$$
\begin{aligned}
\rho\left(A_{u}\right) & \leq \frac{\sqrt{f(v)}}{\sigma_{1}} \\
\left|\sum_{k=1}^{n_{u}} \phi_{k}\right| & \leq \sqrt{f(v)} \sum_{i=1}^{v} \frac{1}{\sigma_{i}}+n_{u}-v
\end{aligned}
$$

and there exists a matrix $Y$ such that

$$
Y-\left(B_{u} B_{u}^{\dagger}-I\right)^{\dagger}\left(B_{u} B_{u}^{\dagger}-I\right) Y \bar{\Lambda} \bar{\Lambda}^{\dagger}>0 .
$$

Proof: Equation (28) follows immediately from the fact that $A_{u}$ and $M \Lambda N$ have the same spectrum and $\rho(M \Lambda N)=$ $\rho(N M \Lambda) \leq\|N M \Lambda\|=\sqrt{f(v)} / \sigma_{1}$. Equation (29) can be proved by noting $\sum_{k=1}^{n_{u}} \phi_{k}=\operatorname{tr}\left(Z^{-1} A_{u} Z\right)=\operatorname{tr}(N M \Lambda)$ and $-\operatorname{tr}(\Lambda) \leq \operatorname{tr}(N M \Lambda) \leq \operatorname{tr}(\Lambda)$, which follows from the diagonal positivity of $\Lambda$ and the orthogonality of $N M$. To prove (30), noting that $P_{u}^{-1}=X_{u}>0$ in Theorem 5 can be decomposed as $X_{u}=Z^{2}$, $Z>0$ without loss of generality, and $Z$ satisfying (24b) can be parameterized as $Y-\left(B_{u} B_{u}^{\dagger}-I\right)^{\dagger}\left(B_{u} B_{u}^{\dagger}-I\right) Y \bar{\Lambda} \bar{\Lambda}^{\dagger}$, where $Y$ is arbitrary, one has the result immediately.

Example 1: Consider a feedback control system with $\phi_{1}=2$, $\phi_{2}=5$, and $\phi_{3}=8$. The channel noise variances are $\sigma_{1}^{2}=0.4$ and $\sigma_{2}^{2}=0.5$. Simple calculations give that $\rho\left(A_{u}\right)=8,\left|\sum_{k=1}^{n_{u}} \phi_{k}\right|=$ $15, \sqrt{f(v)} / \sigma_{1}=3.1623$, and $\sqrt{f(v)} \sum_{i=1}^{v}\left(1 / \sigma_{i}\right)+n_{u}-v=10$. Obviously, (28) and (29) are not satisfied, and hence (24) has no nonsingular solution.

As for dynamic decoders, it is not difficult to show that, through some algebraic manipulations, they are not helpful in reducing the lower bound on transmitted power, and thus unnecessary for stabilization if the lower bound can be achieved by static encoders/decoders. However, dynamic decoders may reduce actual transmitted power if the lower bound cannot be achieved by static ones.

\section{Channel Capacity and Stabilizability}

The information capacity of the discrete-time power constrained parallel Gaussian channel [17] is

$$
\mathcal{C}=\max _{\operatorname{POW}(s) \leq \mathcal{P}} I(s ; r)=\max _{\operatorname{POW}(s) \leq \mathcal{P}} \frac{1}{2} \log _{2} \prod_{i=1}^{m}\left(1+\frac{\mathcal{P}_{i}}{\sigma_{i}^{2}}\right)
$$

where $\mathcal{P}_{i} \geq 0$ is the transmitted power of the $i$ th subchannel, and $\sum_{i=1}^{m} \mathcal{P}_{i} \leq \mathcal{P}$. Noting that, when $\sum_{i=1}^{m} \mathcal{P}_{i}=\mathcal{P}$

$$
\begin{aligned}
\prod_{i=1}^{m}\left(1+\frac{\mathcal{P}_{i}}{\sigma_{i}^{2}}\right) & \leq\left(\prod_{i=1}^{m} \frac{1}{\sigma_{i}^{2}}\right)\left(\frac{\sum_{j=1}^{m}\left(\sigma_{j}^{2}+\mathcal{P}_{j}\right)}{m}\right)^{m} \\
& =\left(\prod_{i=1}^{m} \frac{1}{\sigma_{i}^{2}}\right)\left(\frac{\sum_{j=1}^{m} \sigma_{j}^{2}+\mathcal{P}}{m}\right)^{m}
\end{aligned}
$$

where the first equality holds when $\sigma_{1}^{2}+\mathcal{P}_{1}=\sigma_{2}^{2}+\mathcal{P}_{2}=\cdots=$ $\sigma_{m}^{2}+\mathcal{P}_{m}=\left(\sum_{i=1}^{m} \sigma_{i}^{2}+\mathcal{P}\right) / m$. If $\sigma_{m}^{2} \geq\left(\sum_{i=1}^{m} \sigma_{i}^{2}+\mathcal{P}\right) / m$, then to maximize mutual information, $\mathcal{P}_{m}$ should be set to zero. Likewise, if $\sigma_{m-1}^{2} \geq\left(\sum_{i=1}^{m-1} \sigma_{i}^{2}+\mathcal{P}\right) /(m-1), \mathcal{P}_{m-1}$ should be set to zero. 
Repeat this process until $\sigma_{w}^{2}<\left(\sum_{i=1}^{w} \sigma_{i}^{2}+\mathcal{P}\right) / w$. Then, the optimal power allocation is

$$
\mathcal{P}_{i}^{*}= \begin{cases}\frac{\sum_{j=1}^{w} \sigma_{j}^{2}+\mathcal{P}}{w}-\sigma_{i}^{2} & i \leq w \\ 0 & i>w\end{cases}
$$

and, therefore, the information capacity can be evaluated as

$$
\mathcal{C}=\frac{1}{2} \log _{2} \frac{\left(\mathcal{P}+\sum_{j=1}^{w} \sigma_{j}^{2}\right)^{w}}{\prod_{i=1}^{w} \sigma_{i}^{2}} \quad \frac{\text { bits }}{\text { transmission }} .
$$

The power allocation process for discrete-time parallel Gaussian channels is usually referred to as water filling [17] due to its similarity to distribution of water in a vessel, and is regarded as a major difference from continuous-time channels. Based on this, we have the following result.

Theorem 6: The system in (1), subject to Assumptions 1-3 and power constraint (5), is stabilizable via a pair of encoder $K_{e}$ and decoder $K_{d}$ only if the channel capacity satisfies

$$
\mathcal{C}>\sum_{k=1}^{n_{u}} \log _{2}\left|\phi_{k}\right|+\frac{v}{2} \log _{2} v \triangleq \mathcal{C}_{\text {inf }}
$$

Proof: When the lower bound in (20) is achieved, it is easy to verify that $\sigma_{v}^{2}<\left(\sum_{i=1}^{v} \sigma_{i}^{2}+\mathcal{P}_{i n f}\right) / v$ and $\sigma_{v+1}^{2} \geq$ $\left(\sum_{i=1}^{v+1} \sigma_{i}^{2}+\mathcal{P}_{\text {inf } f}\right) /(v+1)$, where $v$ is defined as in Theorem 4 and $\mathcal{P}_{\text {inf }}=v f(v)-\sum_{j=1}^{v} \sigma_{j}^{2}$. Hence, $w$ in (31) is equal to $v$, and corresponding capacity is $\mathcal{C}_{\text {inf }}$. From this, the result follows immediately. If the lower bound is unachievable, it can be shown readily that corresponding capacity $\mathcal{C}$ is greater than $\mathcal{C}_{\text {inf }}$. This completes the proof.

If the lower bound in (20) is unachievable for certain discrete-time systems, $\mathcal{C}_{\text {inf } f}$ is no longer sufficient for stabilizability, and extra channel capacity would be required. As for the computation of optimal or suboptimal encoders/decoders, one may transform it into a bilinear matrix inequality (BMI) optimization problem by using some techniques developed in $\mathcal{H}_{2}$ control, and we do not intend to include this here due to page length consideration. Further study on computation will be reported elsewhere. The following example shows that, for some systems, the lower bound in (20) is achievable by static LTI encoders/decoders.

Example 2: Consider a system with

$$
A=A_{u}=\left[\begin{array}{ccc}
3 & 0 & 5 \\
-2 & 1 & -1 \\
3 & 2.5 & 1
\end{array}\right], \quad B=B_{u}=\left[\begin{array}{cc}
0.15 & 0.3 \\
-0.3 & 1 \\
0 & -1
\end{array}\right]
$$

The channel noise is characterized by $\Sigma=\operatorname{diag}(2.9,4.8)$. Simple calculations give $v=2, f(2)=410.6400, \mu_{1}^{*}=47.8276$, and $\mu_{2}^{*}=16.8229$. By solving the corresponding BMI, a desired pair of encoder/decoder can be obtained as

$$
\begin{aligned}
& K_{e}=\left[\begin{array}{lll}
-38.5074 & -26.1067 & -40.8811 \\
-39.7752 & -29.2173 & -45.0964
\end{array}\right] \\
& K_{d}=\left[\begin{array}{cc}
0.0374 & -1.4169 \\
-0.6016 & 0.2569
\end{array}\right] \text {. }
\end{aligned}
$$

The actual transmitted power is thus computed as $\operatorname{POW}(s)=$ 789.8300 , which is exactly the same as $v f(v)-\sum_{i=1}^{v} \sigma_{i}^{2}=$ 789.8300. Furthermore, computing transmitted power of each channel gives $\operatorname{POW}\left(s_{1}\right)=402.2300$ and $\operatorname{POW}\left(s_{2}\right)=387.6000$, which are also consistent with the water filling in (31).

\section{CONCLUSION}

We have investigated the state-feedback stabilization problem of multi-input LTI systems over parallel Gaussian channels subject to a total power constraint. It has been shown that to stabilize an LTI plant transmitted power must be greater than a bound related to unstable plant poles and channel noises. In terms of this, lower bounds on required channel capacity for feedback stabilization have been obtained for both continuous-time and discrete-time systems. For continuous-time systems with LTI encoders/decoders, the derived capacity bound is always tight, while, for discrete-time systems with LTI encoders/decoders, the tightness of the capacity bound is not guaranteed. In addition, to achieve the optimal transmitted power, both encoder and decoder in general must be utilized.

\section{REFERENCES}

[1] P. J. Antsaklis and J. Baillieul, "Special issue on networked control," IEEE Trans. Autom. Control, vol. 49, no. 9, pp. 1421-1423, Sep. 2004.

[2] J. Baillieul and P. J. Antsaklis, "Special issue on technology of networked control systems," Proc. IEEE, vol. 95, no. 1, pp. 5-8, Jan. 2007.

[3] G. N. Nair and R. J. Evans, "Stabilization with data-rate-limited feedback: Tightest attainable bounds," Syst. Control Lett., vol. 41, no. 1, pp. 49-56, Sep. 2000.

[4] G. N. Nair and R. J. Evans, "Exponential stabilisability of finite-dimensional linear systems with limited data rates," Automatica, vol. 39, no. 4, pp. 585-593, Apr. 2003.

[5] G. N. Nair and R. J. Evans, "Stabilizability of stochastic linear systems with finite feedback data rates," SIAM J. Control Optim., vol. 43, no. 2, pp. 413-436, 2004.

[6] S. Tatikonda, A. Sahai, and S. Mitter, "Stochastic linear control over a communication channel," IEEE Trans. Autom. Control, vol. 49, no. 9, pp. $1549-1561$, Sep. 2004.

[7] A. S. Matveev and A. V. Savkin, "The problem of LQG optimal control via a limited capacity communication channel," Syst. Control Lett., vol. 53, no. 1, pp. 51-64, Sep. 2004.

[8] A. S. Matveev and A. V. Savkin, "An analogue of Shannon information theory for detection and stabilization via noisy discrete communication channels," SIAM J. Control Optim., vol. 46, no. 4, pp. 1323-1367, 2007.

[9] G. N. Nair, F. Fagnani, S. Zampieri, and R. J. Evans, "Feedback control under data rate constraints: An overview," Proc. IEEE, vol. 95, no. 1, pp. 108-137, Jan. 2007.

[10] J. H. Braslavsky, R. H. Middleton, and J. S. Freudenberg, "Feedback stabilization over signal-to-noise ratio constrained channels," IEEE Trans. Autom. Control, vol. 52, no. 8, pp. 1391-1403, Aug. 2007.

[11] R. H. Middleton, A. J. Rojas, J. S. Freudenberg, and J. H. Braslavsky, "Feedback stabilization over a first order moving average Gaussian noise channel," IEEE Trans. Autom. Control, vol. 54, no. 1, pp. 163-167, Jan. 2009.

[12] J. S. Freudenberg, R. H. Middleton, and V. Solo, "Stabilization and disturbance attenuation over a Gaussian communication channel," IEEE Trans. Autom. Control, vol. 55, no. 3, pp. 795-799, Mar. 2010.

[13] R. W. Brockett and D. Liberzon, "Quantized feedback stabilization of linear systems," IEEE Trans. Autom. Control, vol. 45, no. 7, pp. 1279-1289, Jul. 2000.

[14] J. Xiong and J. Lam, "Stabilization of linear systems over networks with bounded packet loss," Automatica, vol. 43, no. 1, pp. 80-87, Jan. 2007.

[15] A. W. Marshall and I. Olkin, Inequalities: Theory of Majorization and its Applications. New York: Academic, 1979.

[16] K. Zhou, J. C. Doyle, and K. Glover, Robust and Optimal Control. Englewood Cliffs, NJ: Prentice-Hall, 1996.

[17] T. M. Cover and J. A. Thomas, Elements of Information Theory. Hoboken, NJ: Wiley-Interscience, 2006.

[18] A. J. Laub, Matrix Analysis for Scientists \& Engineers. Philadelphia, PA: SIAM, 2005. 